\title{
Design of Waveguides, Bends and Splitters in Photonic Crystal Slabs with Hexagonal Holes in a Triangular Lattice
}

\author{
Cazimir G. Bostan* and René M. de Ridder \\ University of Twente, MESA ${ }^{+}$Research Institute, Integrated Optical MicroSystems (IOMS) group \\ P.O.Box 217,7500AE Enschede, the Netherlands,e-mail: R.M.deRidder@utwente.nl \\ *Now at Honeywell Romania SRL, 15 Oltetului Street, 023817-Bucharest, Romania.
}

\begin{abstract}
Waveguides in photonic crystal slabs (PCS) can be obtained by omitting a row of holes (W1-waveguides). In general the propagation properties in such waveguides suffer from the unavoidable periodic sidewall corrugation caused by the remaining parts of the crystal. The corrugation acts as a Bragg reflector, causing the occurrence of so-called mini stopbands in the transmission of the waveguide. The effect is quite strong in PCS with circular holes, but it can be significantly reduced if correctly oriented hexagonal holes are used. This so-called hexagontype PCS allows the design of waveguides, bends and splitters having a relatively high group velocity and a wide transmission window in the PCS stopband for modes having their magnetic field oriented mainly perpendicular to the slab.
\end{abstract}

Keywords: photonic crystal slab, line defect waveguide, waveguide bend, Y-junction, hexagonal holes.

\section{INTRODUCTION}

Intense research on photonic crystals ( $\mathrm{PhC}$ 's) has been going on during the last decade. In particular, photonic crystal slabs (PCS) have received considerable interest because they have the potential to serve as an integration platform for densely packed photonic circuits.

Until now, theoretical and experimental studies on PCS have almost exclusively concentrated on a triangular lattice of circular holes. The 'defect-free' air bridge slab has a large robust gap in H-like modes (having their magnetic field vectors perpendicular to the slab at its mid-plane) between the first two bands [1]. Hexagonal holes provide the same large gap as their circular counterpart and also add some flexibility in design. An extra degree of freedom is the rotation angle of the hexagons with respect to their symmetry axis (see Fig. 1). This can be used as tuning means for adjusting the configuration of the lattice (i.e. size and orientation of veins and islands). By increasing the filling factor one can obtain absolute gaps in guided modes [2], situated at higher frequencies in the band diagram.

In a previous paper [3] we have shown that the guided modes in a line defect can be tuned by combining hexagonal holes with triangular air inclusions (see Fig. 3). These inclusions can be used to reduce the waveguide width (hence the effective index of the waveguide) independently of the lattice constant and without lattice distortion, and also to minimise the effect of boundary corrugations. Hexagonal holes, in combination with triangular air inclusions can make linear defect waveguides (bandgap-guided in the slab plane) that closely resemble ridge waveguides. The resulting waveguide shows single-mode behaviour, with a high group velocity and a relatively large transmission window.

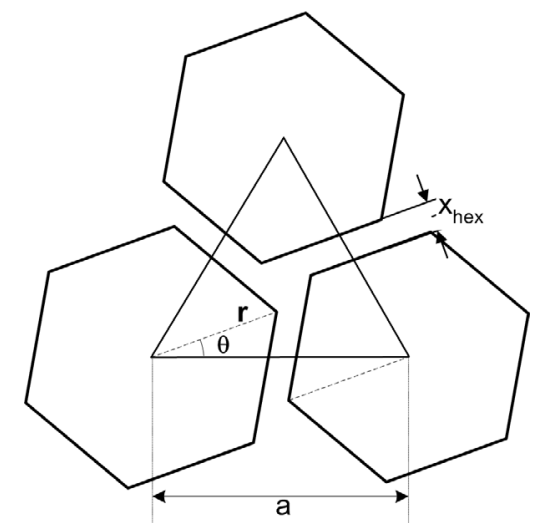

Figure 1. Hexagon-type PhC configuration - rotated hexagons arranged in a triangular lattice (for $\theta=0$, the vertices of neighbouring hexagons are pointing towards each other, the rotation is taken counter-clockwise); geometrical parameters are indicated: lattice constant (a), radius (r), rotation angle $(\theta)$, vein thickness (xhex).

This work was supported by the Dutch Technology Foundation STW and the MESA ${ }^{+}$SRO Advanced Photonic Structures. 
In this paper we consider further developments of these line defect waveguides, namely combining them to form bends and Y-junctions. The bends and Y-junctions include intermediate short waveguide sections at half the bend angle playing the role of corner 'mirrors'. For hexagons with $\theta=30^{\circ}$ rotation angle, the inner edge of these waveguide sections is straight, and this opens the possibility to make them resemble ridge waveguides, too (see Figs. $5 \& 6$ ). Then, one can achieve good mode matching between input and output branches and intermediate sections.

\section{DESIGN GUIDELINES}

Line defect waveguides can be combined to form bends and branches. Connecting two or three line defect waveguides at an angle (e.g. $60^{\circ}$ in a triangular lattice) makes a bend or a Y-junction, respectively. The main role of the $\mathrm{PhC}$ is to prohibit the in-plane bend losses, and allow curvature radii as small as the wavelength. The translational symmetry of straight waveguide is broken when introducing a bend or a Y-junction; then, even and odd modes can couple to each other. The following phenomena that occur at a normal bend are detrimental to the transmission: (a) strong back-reflections; (b) increased out-of-plane losses; (c) relatively poor matching between the guided modes of the straight sections (because the bend is usually multimode). On the other hand, the transmission along a bend can be higher than in a line defect waveguide, in the mini stopbands of the latter.

Several designs of bends and Y-junctions have been proposed, targeting minimisation of back-reflections and improvement of mode-matching: (1) place small holes in the centre of constituent W1 waveguides [4] or at the connection point of a Y-junction [5]; (2) displace holes from the inner to the outer corner of the bend [6]; (3) introduce an intermediate short section at half of the bend angle [6], [7]; (4) reduce the corner angle in a polycrystalline $\mathrm{PhC}$ made by dislocating lattice blocks [8]; (5) deform the lattice in and around the bend [9]; (6) modify the lattice topology by connecting several holes at the corner [10].

Resonant elements incorporated in the waveguide (e.g. displaced holes that act as resonators) can give an increased transmission for certain wavelengths but, most of the time, do not provide a large bandwidth. Lattice deformation can be effective, but then the concept of $\mathrm{PhC}$ is somewhat obscured. In a naïve ray picture, an intermediate short section at half of the bend angle acts as a corner mirror that directs an incident ray from the input to the output branch of a bend. This intuitive argument proved to be effective [6],[7], therefore we apply it in our designs, aiming also at keeping the resemblance with ridge waveguides. When using $\theta=30^{\circ}$, the inner edge of a short intermediate waveguide section at $30^{\circ}$ appears naturally, without the need to change any hole. Some modifications of the $\mathrm{PhC}$ structure are needed to obtain the proper outer edge of the intermediate waveguide section. For a bend, half of the hexagonal corner hole is filled and the hole-shape is changed into a trapezium (Fig. 5b). For a Y-junction, an extra equilateral triangle is introduced at the connection point (Fig. 6b).

\section{SIMULATION}

\subsection{Effective index approximation and computational method}

Rigorous modeling of PCS structures requires 3D calculations that are very demanding in terms of time and memory requirements. An effective index approximation of PCS has been used for relieving these requirements by reducing the full 3D calculations to simpler, though approximate $2 \mathrm{D}$ calculations. The PCS is essentially a slab system perforated with a lattice of holes. In the effective index method, the PCS is replaced by a $2 \mathrm{D}$ system with the background dielectric medium having the effective refractive index of the fundamental guided mode of the slab system. In high-index contrast systems (like SOI) the effective index approximation is valid for a narrow frequency range [11] in which the guided mode dispersion is small enough. In ref. [3] we have applied the effective index approximation for qualitative design of 3D photonic crystal structures (slabs and line defect waveguides). Although the bands calculated using the effective index approximation are shifted towards lower frequencies compared to those of the real 3D structure, the shape of the lowest order bands is roughly preserved, allowing conclusions regarding the classification of modes in 3D. Qualitative design rules can be obtained from a $2 \mathrm{D}$ calculation combined with the light-line of a cladding that restricts the $\omega-k$ range available for guided modes in the actual 3D case.

We choose a slab system (Fig. 2) in which the bottom cladding is a quasi-2 $\mathrm{D}_{\mathrm{SiO}_{2}}$ slab (i.e. the air holes penetrating both $\mathrm{Si}$ and $\mathrm{SiO}_{2}$ layers of a SOI wafer). Etching the air holes into the bottom cladding has beneficial effects: it decreases the asymmetry and increases the frequency range below the light cone, available for truly guided modes. Moreover, the structure is mechanically stable and the etch depth can be moderate because of the good confinement provided by the high-index contrast. To avoid complications due to possible coupling to higher order modes, we choose a slab system that supports only a single mode, for both polarisations. 

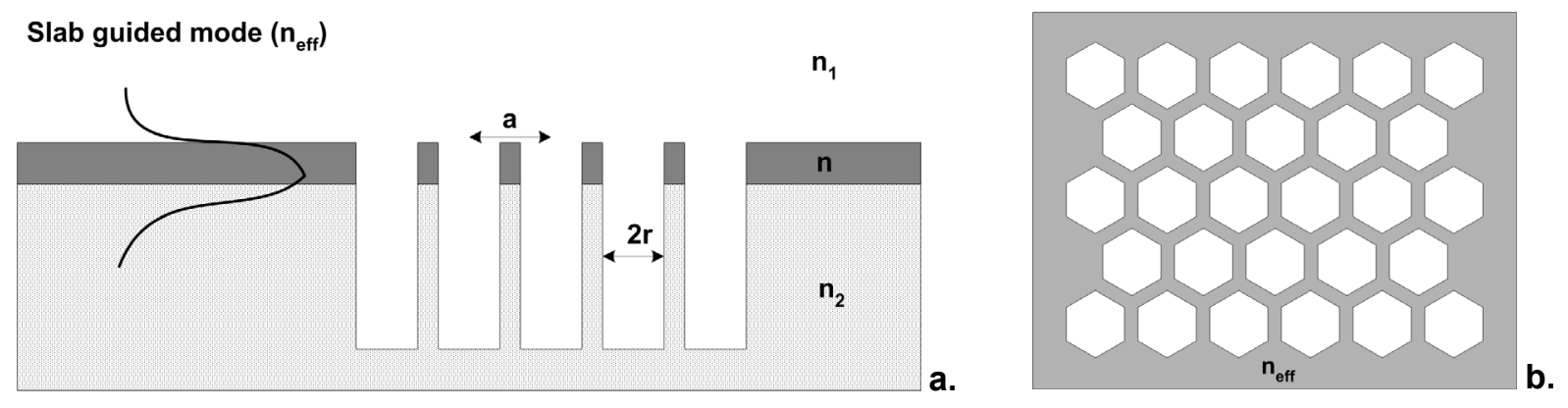

Figure 2 Illustration of the effective index approximation.

The single mode condition of an asymmetric slab system consisting of a core with refractive index 3.45 ( $\mathrm{Si}$ ), sandwiched between two claddings with refractive indices 1.0 (air) and $1.45\left(\mathrm{SiO}_{2}\right)$ at a free-space wavelength $\lambda_{0}=1.55 \mu \mathrm{m}$ can be calculated to result in a maximum core thickness of $273 \mathrm{~nm}$. We choose this thickness to be $h=260 \mathrm{~nm}$. The effective indices of TE and TM-polarised guided slab modes will then be $n_{\text {eff, TE }}=2.924$ and $n_{\text {eff, TM }}=2.24$. For a 2D PhC system, the TE/TM notation is somewhat awkward, hence, modes having their magnetic field parallel to the infinite dimension are denoted as H-modes, while those with the electric field vector along the infinite dimension are called E-modes. In the effective index approximation, the direction perpendicular to the slab is extended to infinity, so H-type modes have to be calculated using the effective index for TE slab modes. There is no $2 \mathrm{D}$ gap in E-modes for $n_{\text {eff, } \mathrm{TM}}=2.24$, therefore we focus only on the $\mathrm{H}$ modes.

The projected band diagrams of line defect waveguides have been calculated using the MIT Photonic Bands program [12]. We used a supercell with the central line defect of length $a$ (the periodicity along $\Gamma$-K) and 8 rows of holes wide (Fig. 3a). Bloch boundary conditions were applied at the sides of the supercell.

The behaviour of finite-sized samples used in actual experiments cannot be readily expressed in terms of Bloch modes and an important role is played by evanescent modes and reflections from the boundaries. Therefore, we calculated the spectral transmittance using 2D finite-element (FEM) simulations using FEMLAB [13]. The FEM has the advantage of outputting the stationary field profiles, so that the field energy localisation and the local direction of power flow can be easily calculated. Although the material dispersion is neglected here (assumption valid for $\mathrm{Si}$ and $\mathrm{SiO}_{2}$ at the working wavelengths), this can be easily accounted for in the FEM, which is a frequency-domain method. The computational domain is finite, with transparent ('low reflecting') boundary conditions. At the waveguide input a constant amplitude field is applied. Power outflow is monitored at the other end of the waveguide. Transmittance is given by the ratio between the power outflow at the output boundary and power inflow at the input boundary (it is influenced by the coupling of the input field with the waveguide modes). Normalised frequency $(u=a / \lambda)$ is scanned in the gap range, with a small step.

\subsection{Results}

We consider a waveguide along the $\Gamma-\mathrm{K}$ direction with a central symmetry axis and lattice orientation $\theta=30^{\circ}$; the lattice is undistorted and air inclusions are isosceles triangles with $120^{\circ}$ apex angle and equal sides $1.1 a$ (see Fig. 3a).

The band diagram of the original, perfectly periodic lattice was projected along the $\Gamma-\mathrm{K}$ direction, revealing continua of states that extend in the bulk photonic crystal (grey regions in Fig. 3b) and the projected gap opened between them. The isolated dispersion curves that appear in the projected gap correspond to states localised in the line defect. The light line (straight solid line) corresponds to an effective index of the bottom cladding $n_{\text {eff, } \mathrm{SiO} 2}=1.238$.

We focus our attention on the even mode in Fig. 3b. This is in fact the fold-back of the index-guided mode at the edge of the line defect Brillouin zone. This mode undergoes an anticrossing with another even mode from the lower edge of the gap (around $k=0.36$ ). As a consequence, the spectral region below the light line of the even mode is very limited $(u=0.284 \ldots 0.29)$ and its group velocity is small. However, the even mode covers a broad range of frequencies above the light line. Even for modes situated above the light line the propagation losses might be low in practice. For $u=0.29 \ldots 0.326$ there is a single mode region, covered by the even mode that has a high group velocity $\left(v_{g}=0.31 c\right)$.

An important parameter is the position of the triangles with respect to the waveguide's central axis $\left(x_{t}\right)$. The influence of $x_{t}$ on the band diagram has been assessed through a series of simulations. An example of computed band diagram, for $x_{t}=1 / 2 r \sqrt{3}$, is shown in Fig. $3 \mathrm{~b}$. When $x_{t}$ increases, the following changes in the band diagram take place: (a) the odd mode shifts upwards; (b) the even mode shifts downwards; (c) the anticrossing decreases and shifts to lower $k$ values. 

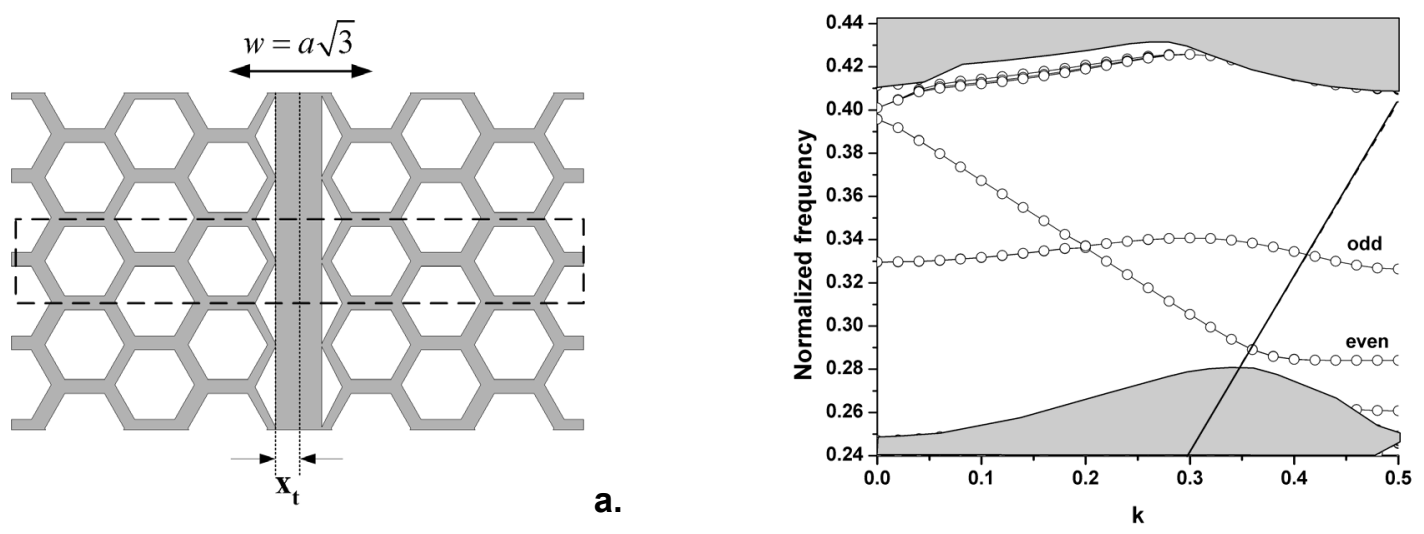

b.

Figure 3. Line defect waveguide: $\boldsymbol{a}$. - domain geometry and supercell (dashed line); $\boldsymbol{b}$. - band diagram of guided modes (grey regions are bulk modes; the light line is solid); $x_{t}=r \sqrt{3} / 2$.

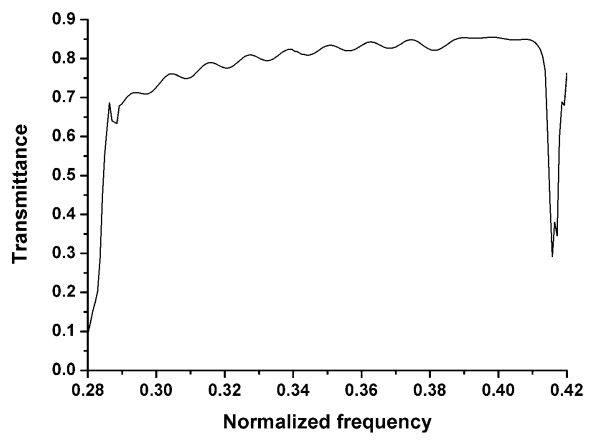

a.

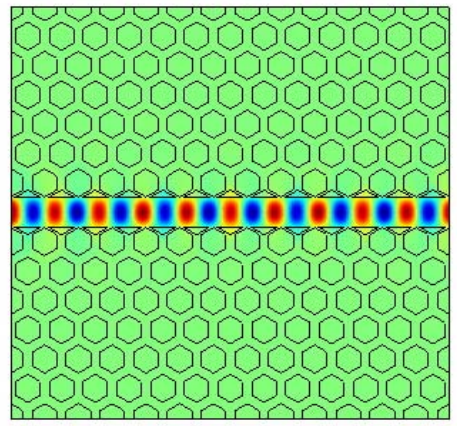

b.

Figure 4. a. - spectral transmittance through a line defect waveguide (see text for details); b. - the H field distribution at normalised frequency $u=0.315$.

The spectral transmittance through the line defect waveguide, bend and Y-junction was calculated by FEM for $u=0.28 \ldots 0.42$ with 200 steps.

The transmittance through the line defect waveguide (Fig. 4a) has a broad band, with a small ripple. The maximum transmittance is about $85 \%$, because the coupling between the input source and the mode guided in the line-defect waveguide was not optimised. The field distribution for an H-mode at normalised frequency $u=0.315$ is shown in Fig. $4 \mathrm{~b}$. The field profile clearly resembles that of an index-guided mode. The transmittance through the line defect waveguide is considered as a reference for assessing the efficiency of bends and Y-junctions. We define the relative transmittance $\left(T_{r}\right)$ as the ratio between the transmittance through the bend or $\mathrm{Y}$-junction and the transmittance through the straight line-defect waveguide.

We consider a bend (Fig. 5) and a Y-junction (Fig. 6) with almost the same length as the line-defect waveguide. For the bend, $T_{r}>89 \%$ in the range $u=0.302 . .0 .316$ (Fig. 5a). For the Y-junction, $T_{r}>63 \%$ in the range $u=0.302 . .0 .32$ (Fig. 6a).
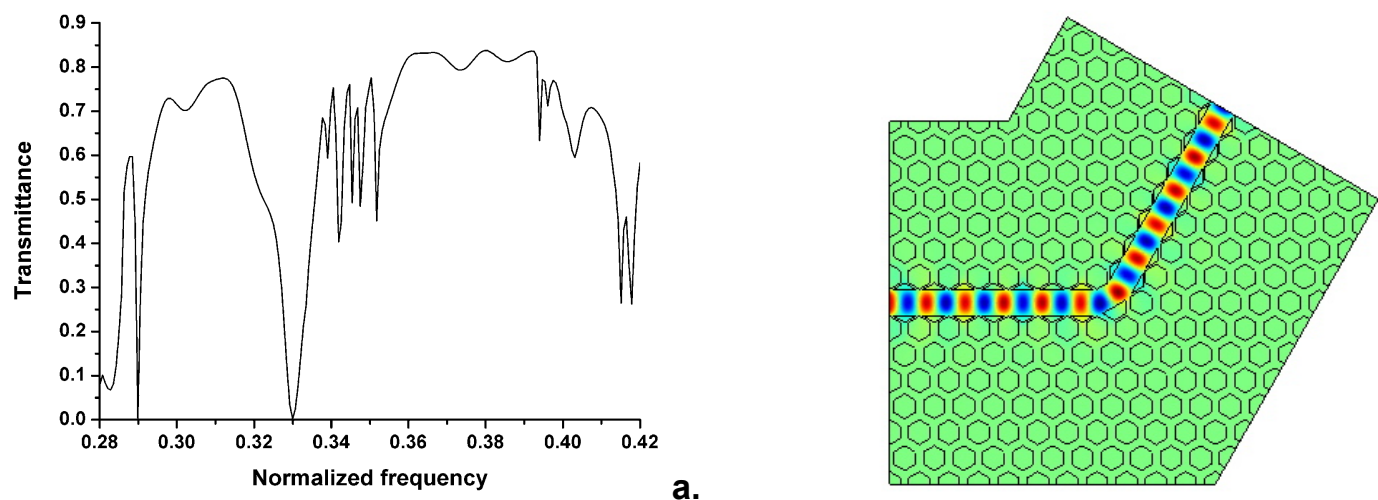

a.

b.

Figure 5. a. - spectral transmittance through a bend;

b. - the H field distribution at normalised frequency $u=0.315$. 

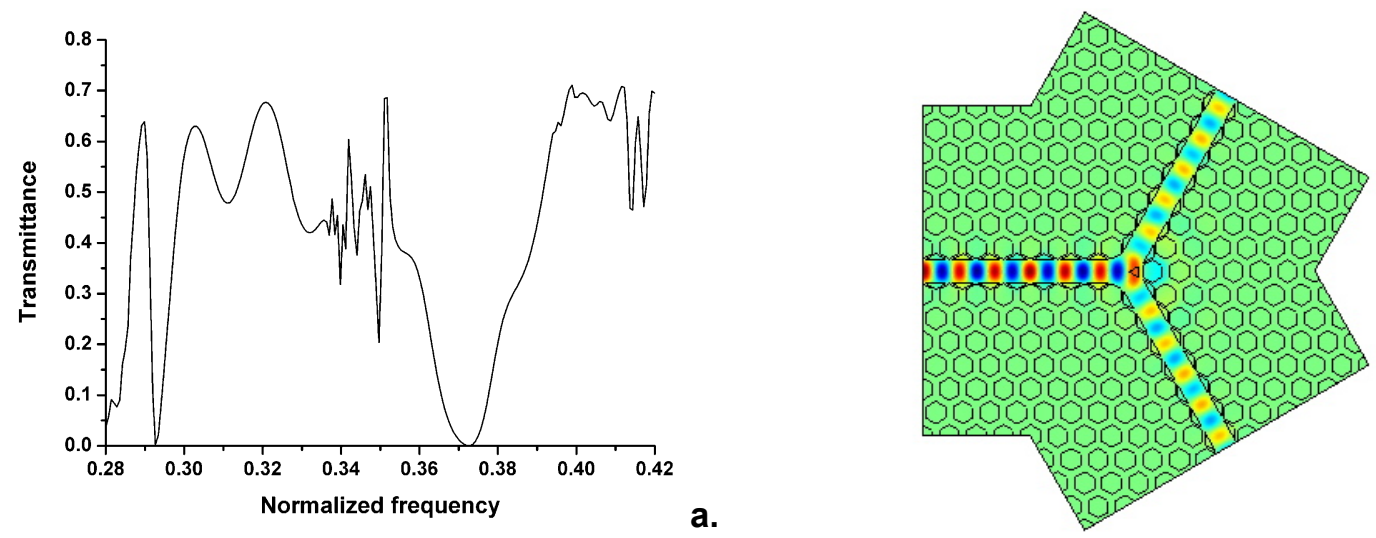

b.

Figure 6. a. - total spectral transmittance through a Y-junction (sum of the two branches);

b. - the H field distribution at normalised frequency $u=0.315$.

Both the bend and the $\mathrm{Y}$-junction are characterised by resonances of the corner cavities $(u=0.33$ for the bend; $u=0.373$ for the Y-junction). Then light is 'trapped' at the corner and the transmittance is close to zero. The separation in even and odd modes is not possible anymore in a bend or Y-junction, and this leads to mode mixing. The mixing between two modes can be seen in the transmittance, in the form of rapidly oscillating spikes in the range $u=0.338 . .0 .352$ (Figs. $5 \mathrm{~b} \& 6 \mathrm{~b}$ ).

\section{DISCUSSION AND CONCLUSIONS}

We presented a design approach for line-defect waveguides in 'hexagon-type' PCS, based on a novel way of tuning 'defect' modes by means of triangular air inclusions. The size and positions of these inclusions modify the effective index of the waveguide independently of the lattice constant (in some cases without lattice distortion) and also minimise the effect of boundary corrugations. The waveguides are integrated in a hexagontype PCS and they have the following characteristics: reduced DBR effect, single-mode, high group velocity and large transmission window. These waveguides, closely resembling conventional ridge waveguides, can be combined to form efficient bends and Y-junctions. The bends and Y-junctions include intermediate short waveguide sections at half the bend angle playing the role of corner 'mirrors'. For hexagons with $\theta=30^{\circ}$ rotation angle, the inner edge of these waveguide sections is straight, and this opens the possibility to make them resemble ridge waveguides, too. In this way, one can achieve good mode matching among input and output branches and intermediate sections. PhC's may be a good way for obtaining small high-Q resonators or sensing elements, which should be coupled to the outside world through waveguides. For combining several of these basic devices, possibly in MZI-configurations, bends and Y-junctions are needed. In principle, these could be made almost as small using conventional high-index waveguides. An important reason for implementing them with $\mathrm{PhC}$ waveguides is the problem of matching conventional waveguides to $\mathrm{PhC}$ waveguides (probably a lot of those transitions result in larger overall loss and larger size than implementing the entire circuit in $\mathrm{PhC}$ ). In this case the coupling efficiency with input the access ridge waveguides may play a more prominent role than radiation losses. One has also to consider the wavelength dependency of the transmission, due to DBR and Fabry-Perot effects (these depend on the length of line defect arms). The effective index method, allowing for efficient 2D calculations, gives qualitative results, which could be used for a first design. Fine-tuning through $3 \mathrm{D}$ calculations is necessary to account for out-of-plane losses.

It is worth noting that we have considered only H-modes and it seems very hard to obtain polarisationindependent functioning in these devices.

\section{REFERENCES}

[1] S.G. Johnson, S.H. Fan, P.R. Villeneuve, J.D. Joannopoulos, and L.A. Kolodziejski: Guided modes in photonic crystal slabs, Phys. Rev. B, vol. 60, pp. 5751-5758, 1999.

[2] C.G. Bostan and R.M de Ridder: Design of photonic crystal slab structures with absolute gaps in guided modes, J. Opt. Adv. Mat., vol. 4, pp. 921-928, 2002.

[3] C.G. Bostan, R.M. de Ridder, V.J. Gadgil, H. Kelderman, L. Kuipers, and A. Driessen: Design and fabrication of line-defect waveguides in hexagon-type SOI photonic crystal slabs, Photonic Crystal Materials and Nanostructures - Photonics Europe 2004, Strasbourg - France, ed. R.M. De La Rue et al., in Proc. SPIE, vol. 5450, pp. 323-332, 2004.

[4] A. Chutinan, M. Okano, and S. Noda: Wider bandwidth with high transmission through waveguide bends in two-dimensional photonic crystal slabs, Appl. Phys. Lett., vol. 80, pp. 1698-1700, 2002. 
[5] S. Boscolo, M. Midrio, and T.F. Krauss: Y junctions in photonic crystal channel waveguides: high transmission and impedance matching, Opt. Lett., vol. 27, pp. 1001-1003, 2002.

[6] H. Benisty, S. Olivier, C. Weisbuch, M. Agio, M. Kafesaki, C.M. Soukoulis, M. Qiu, M. Swillo, A. Karlsson, B. Jaskorzynska, A. Talneau, J.Moosburger, M. Kamp, A. Forchel, R. Ferrini, R. Houdre and U. Oesterle: Models and measurements for the transmission of submicron-width waveguide bends defined in two-dimensional photonic crystals, IEEE J. Quantum Electron., vol. 38, pp. 770-785, 2002.

[7] A. Mekis, J.C. Chen, I. Kurland, S.H. Fan, P.R. Villeneuve, and J.D. Joannopoulos: High transmission through sharp bends in photonic crystal waveguides, Phys. Rev. Lett., vol. 77, pp. 3787-3790, 1996.

[8] A. Sharkawy, D. Pustai, S.Y. Shi, and D.W. Prather: High transmission through waveguide bends by use of polycrystalline photonic-crystal structures, Opt. Lett., vol. 28, pp. 1197-1199, 2003.

[9] I. Ntakis, P. Pottier, and R.M. De La Rue: Optimization of transmission properties of two-dimensional photonic crystal channel waveguide bends through local lattice deformation, J. Appl. Phys., vol. 96, pp. 12-18, 2004.

[10] J.S. Jensen and O. Sigmund: Systematic design of photonic crystal structures using topology optimization: Low-loss waveguide bends, Appl. Phys. Lett., vol. 84, pp. 2022-2024, 2004.

[11] M. Qiu: Effective index method for heterostructure-slab-waveguide-based two-dimensional photonic crystals, Appl. Phys. Lett., vol. 81, pp. 1163-1165, 2002.

[12] S.G. Johnson and J.D. Joannopoulos: Block-iterative frequency-domain methods for Maxwell's equations in a planewave basis, Opt. Express, vol. 8, pp.173-190, 2001.

[13] FEMLAB is a trademark of Comsol AB, Sweden, http://www.femlab.com. 